\title{
Comparaison de quelques caractéristiques de la turbulence mesurées dans divers écoulements d'eau en canal et en conduite
}

\author{
Félix Raemy \\ Ecole polytechnique fédérale de Zurich
}

\section{Introduction}

La description détaillée de nombreux phénomènes tels que la diffusion, la dissipation d'énergie ou le transport des sédiments repose sur la connaissance de la structure turbulente de l'écoulement concerné. Parallèlement à l'évolution des techniques de mesure, une intense recherche expérimentale s'est développée depuis une quarantaine d'années dans ce domaine. Très tôt, les anémomètres à fil chaud ont été utilisés pour la détermination des caractéristiques de la turbulence dans l'air. L'extension à l'étude des écoulements d'eau s'est par contre avérée plus délicate. Seule l'apparition, il y a environ vingt-cinq ans, des sondes quartzées à film chaud et plus récemment celle des anémomètres à laser ont permis des progrès importants. D'autres méthodes moins répandues (visualisation par bulles d'hydrogène, vélocimètres utilisant le principe du tube de Prandtl) ont également été appliquées avec succès. Alors que dans l'air une information dense a été acquise tant en conduite lisse que rugueuse (LAUFER 1954 [9], ROBERTSON et al. 1965 [18], MUÑOZ Goma et Gelhar 1968 [10], Clark 1968 [4], Townes et al. 1972 [21]), les mesures de turbulence dans l'eau se limitent essentiellement aux canaux découverts à paroi lisse ou rugueuse (RAICHLEN 1967 [15], RICHARDSON et MC QUIVEY 1968 [17], Grass 1971 [7], BLINCO et SimONS 1974 [2], NEZU 1977 [11], NEZU et Rodi 1986 [12], CARdoso et al. 1989 [3], WANG et QIAN 1989 [22]) respectivement aux conduites à paroi lisse (RESCH 1968 [16], JohansSon et ALFREDSSON 1982 [8]). Dans le but de combler une lacune existante, l'auteur de cette contribution a présenté en 1978 quelques caractéristiques de la turbulence déterminées dans un écoulement d'eau en conduite à paroi rugueuse. Ces mesures, réalisées par anémométrie à film chaud à des nombres de Reynolds élevés atteignant $3.4 \cdot 10^{5}$, avaient nécessité une mise au point minutieuse de la méthode et la prise de multiples précautions quant à la qualité de l'eau dans l'installation (RAEMY 1978 [14]). Les résultats obtenus s'étaient avérés être en bonne harmonie avec ceux des travaux antérieurs. Depuis lors, la connaissance de la turbulence dans l'eau a pu être largement consolidée et étendue. Il paraît ainsi intéressant d'inclure dans cette comparaison les observations tirées des études entreprises depuis 1978.

\section{Comparison of turbulence characteristics measured in pipe and open-channel water flows}

Some turbulence characteristics which were determined in water flow through a rough pipe by hot-film anemometry [14] are compared with those obtained more recently by other authors using actual measuring techniques. It can be observed that the distributions of the turbulent intensities, the probability density of the fluctuating velocity, the eddy viscosity and of the Taylor microscales are generally in good agreement for pipe and open-channel water flows. Thus, the results of the rough pipe investigation [14] are confirmed. 


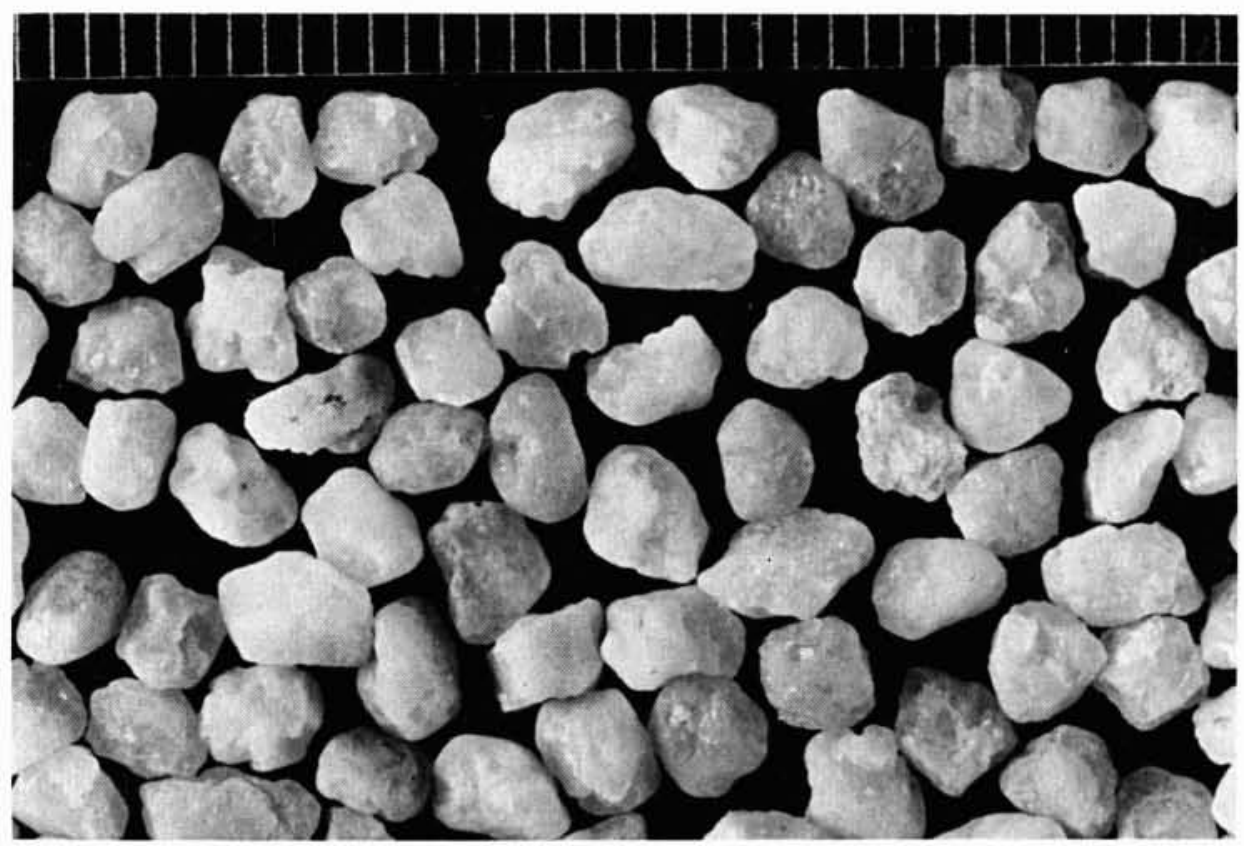

1. Grains de quartz avant collage dans la conduite. Echelle en $\mathrm{mm}$ (diamètre moyen : $3,19 \mathrm{~mm}$ ).

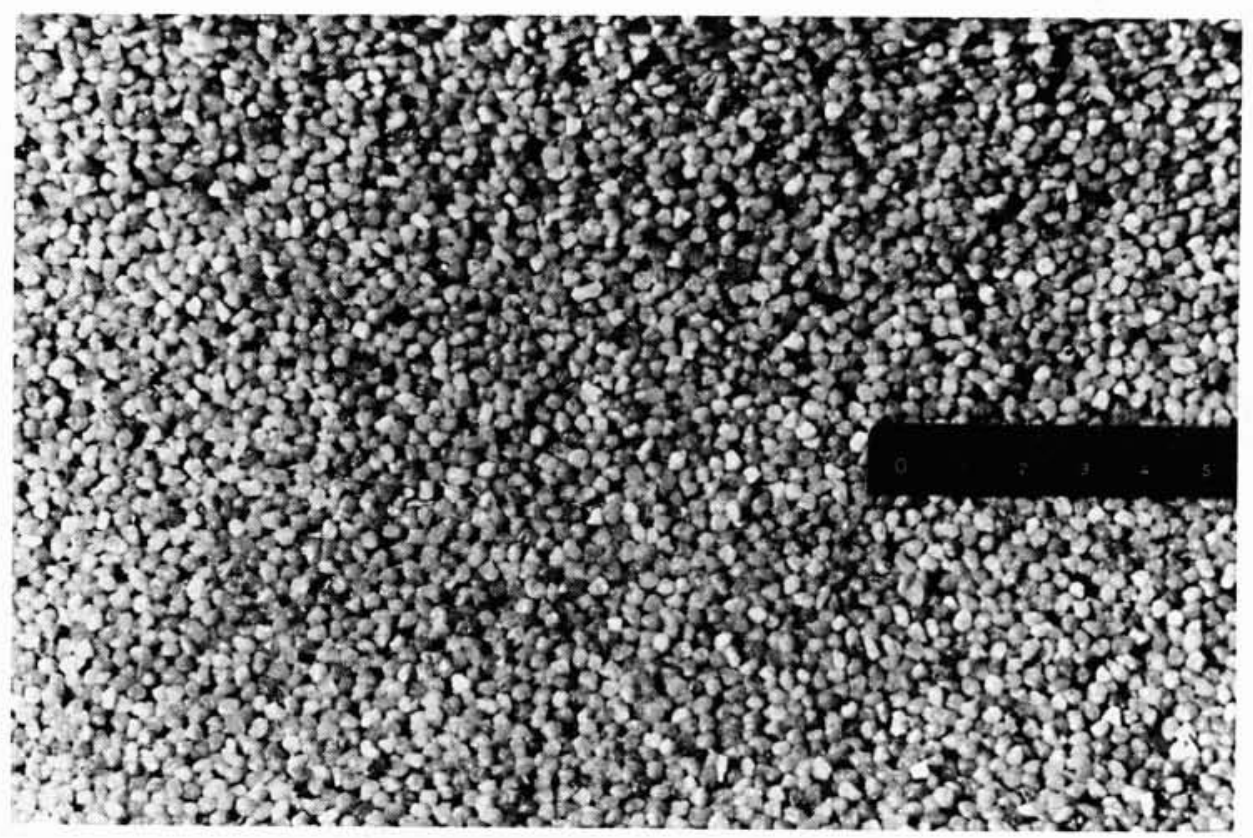

2. Détail de la paroi rugueuse formée d'une couche compacte de grains. Echelle en $\mathrm{cm}$. 


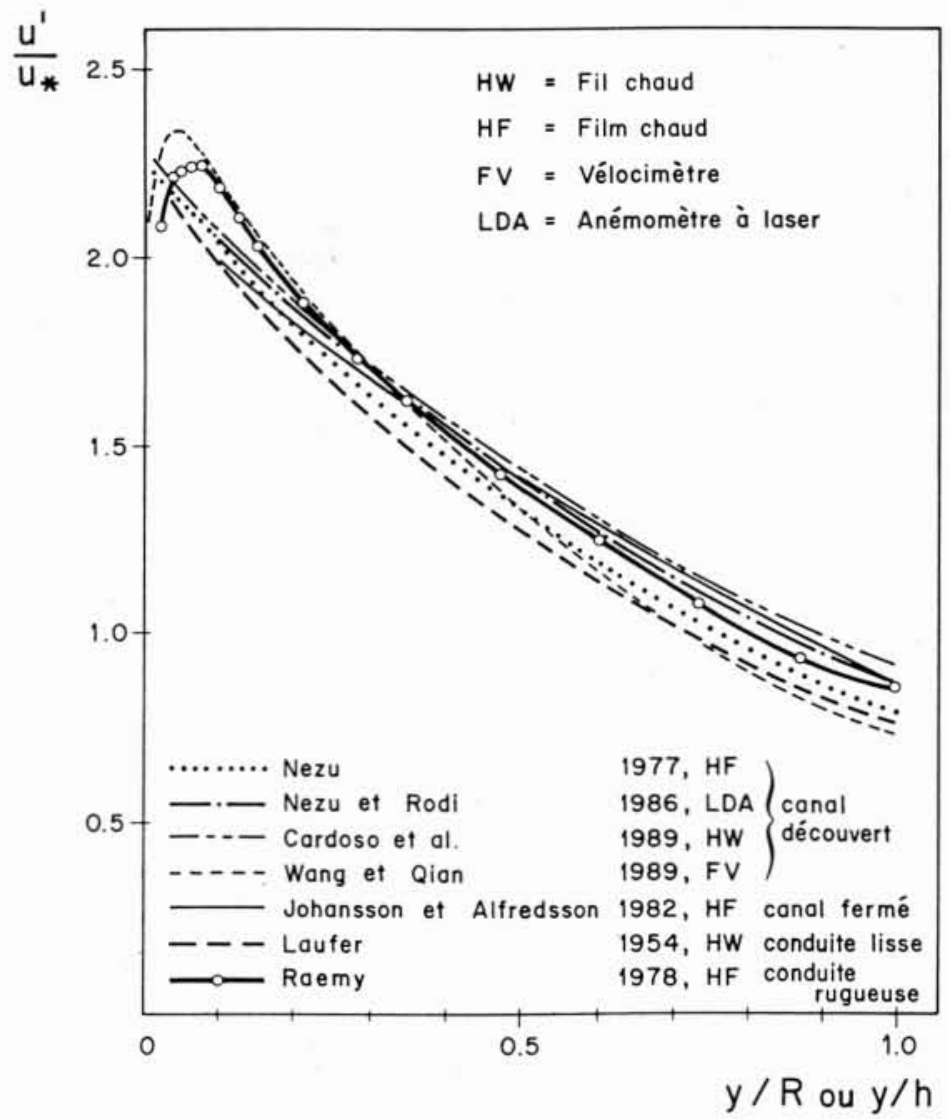

3. Répartition des intensités turbulentes longitudinales $u^{\prime} / u$.

\section{Turbulence dans un écoulement d'eau en conduite à paroi rugueuse}

Un bref aperçu de ce travail est donné ici, des informations plus amples pouvant être trouvées en référence [14]. Les valeurs instantanées de la composante axiale de la vitesse ont été mesurées à l'aide de sondes à film chaud coniques TSI dans une conduite circulaire à paroi recouverte de grains de quartz naturels $\left(d_{m}=3,19 \mathrm{~mm}\right)$ placée dans une soufflerie à eau en circuit fermé (fig. 1 et 2). Avec un diamètre moyen $D_{m}=0,157 \mathrm{~m}$, la conduite rugueuse étudiée présentait une rugosité relative $R_{m} / k_{s}=22,7$, où $R_{m}=D_{m} / 2$ et $k_{s}$ est la hauteur d'aspérité équivalente selon Nikuradse.

Les mesures ont été effectuées dans une section de la conduite située à $70 \cdot D_{m}$ de l'entrée, soit un écoulement pleinement développé, avec $\operatorname{Re}$ variant entre $0,85 \cdot 10^{5}$ et $3,41 \cdot 10^{6}$. Le signal turbulent, transmis par un anémomètre DISA à température constante relié à un linéarisateur, a été traité numériquement sur ordinateur. Les grandeurs classiques suivantes ont été déterminées :

- répartition des vitesses moyennes locales (loi de paroi et de la zone externe) ;

- répartition des intensités turbulentes;

- densités de probabilité de la vitesse et moments d’ordre supérieur ;
- répartition de la viscosité turbulente;

- densités spectrales de puissance (par la méthode de la transformée de Fourier rapide TFR);

- microéchelles de Taylor et dissipation de l'énergie turbulente.

Dans ce qui suit, seules quelques-unes de ces grandeurs seront comparées à celles obtenues ultérieurement par d'autres auteurs.

\section{Intensités turbulentes}

Les intensités turbulentes relatives $u^{\prime} / u$. déterminées dans la conduite rugueuse sont présentées en fonction de $y / R$ sur la figure 3 , où $u^{\prime}$ est l'écart-type de la fluctuation $u$ de la vitesse, $u$. la vitesse de frottement, $y$ la distance à la paroi et $R$ le rayon de la conduite rapporté à l'origine des $y$ (paroi virtuelle). La répartition donnée (RAEMY [14]) est indépendante de Re. Les résultats obtenus dans l'air en conduite lisse par LAUFER [9], respectivement dans l'eau en canal découvert par NEZU [11], NEzU et RODI [12], CARDoso et al. [3], WANG et QIAN [22], en conduite à section rectangulaire par JOHANSSON et ALFREDSSON [8] sont reportés pour comparaison. Dans l'expression $y / h, h$ représente la profondeur d'écoulement. 


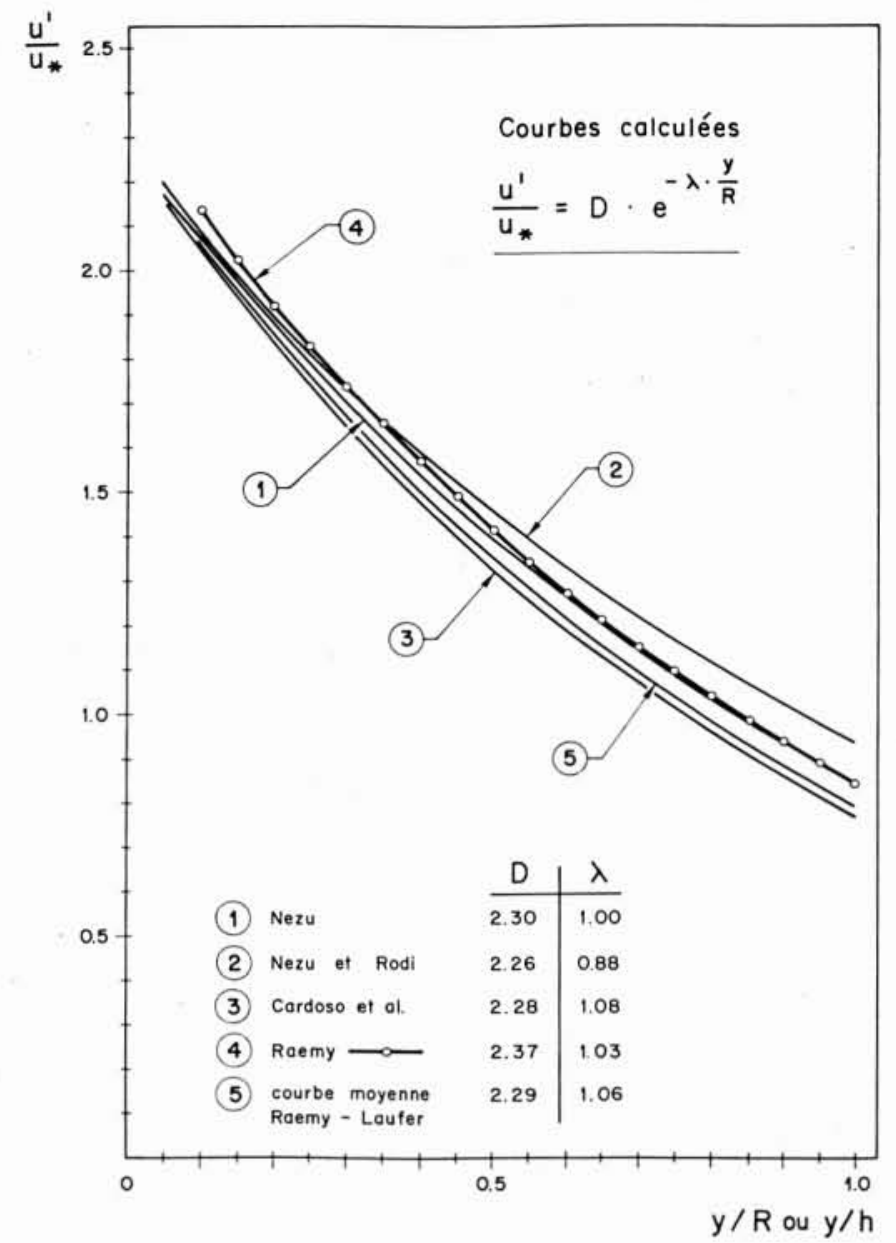

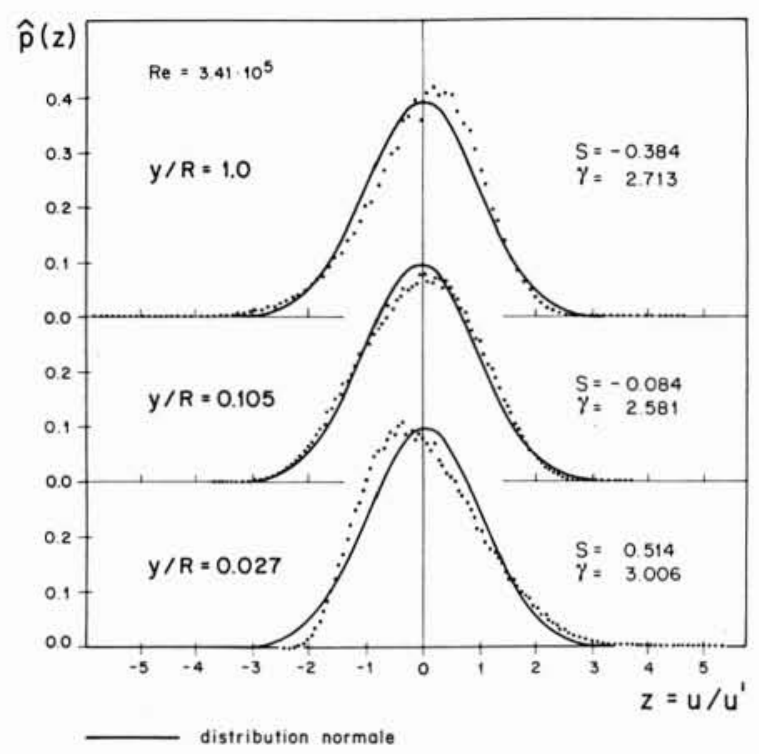

5. Densités de probabilité des fluctuations longitudinales de la vitesse dans la conduite rugueuse.
Malgré la diversité des cas étudiés et celle des méthodes d'investigation utilisées, une concordance frappante des répartitions est visible à $y / R$ ou $y / h \geqslant 0,20$ environ. Au voisinage immédiat de la paroi, $u^{\prime} / u$. semble par contre dépendre de la nature de cette dernière.

Reconnaissant le caractère " universel " de la réparti-

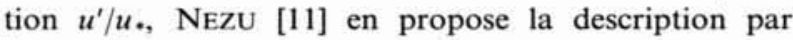
l'expression

$$
\frac{u^{\prime}}{u \cdot}=D \cdot \mathrm{e}^{-\lambda \cdot \frac{y}{h}}
$$

où $D$ et $\lambda$ sont des constantes empiriques. De l'analyse de ses propres résultats, il détermine les valeurs $D=2,30$ et $\lambda=1,0$. Plus tard, NeZU et Rodi [12] tirent de leurs mesures et des travaux réalisés par CLARK [4], Steffler et al. [20], Blinco et PARtheniades [1] les constantes $D=2,26$ et $\lambda=1,08$. En réexaminant l'ensemble des intensités turbulentes déterminées dans la conduite rugueuse [14], on obtient pour la répartition $u^{\prime} / u$. dans le profil de mesure, traitée par la méthode de régression des moindres carrés, les valeurs $D=2,37$ et $\lambda=1,03$. Cette répartition coïncide de manière satisfaisante avec celle donnée par NEZU (fig. 4). D'autre part, elle suit une courbe parallèle à celle indiquée par LAUFER [9] en conduite lisse (fig. 3), dont le travail constitue une référence en littérature. Avec $D=2,29$ et $\lambda=1,06$, la courbe moyenne prise entre les répartitions de LAUFER et de RAEMY (voir fig. 4, courbe 5) peut ainsi être utilisée pour évaluer les intensités $u^{\prime}$ tant sur fond lisse que fortement rugueux. Compte tenu de la dispersion liée à la précision des différentes méthodes de mesure, les courbes de la figure 4 montrent entre elles une bonne cohérence.

\section{Densités de probabilité de la vitesse et moments d'ordre supérieur}

Les densités de probabilité normalisées $\hat{p}(z)$ des fluctuations longitudinales $u$ de la vitesse, où $z=u / u^{\prime}$, ont été déterminées numériquement à différents $\mathrm{Re}$ dans la section de la conduite rugueuse. L'asymétrie $s$ (moment du $3^{\mathrm{e}}$ ordre) et la kurtosis $\gamma$ (moment du $4^{\mathrm{e}}$ ordre) de leur distribution ont également été calculées, avec $s=\bar{u}^{3} / u^{\prime 3}$ et $\gamma=\bar{u}^{4} / u^{\prime 4}$. La figure 5 montre l'allure des distributions obtenues pour $\operatorname{Re}=3,41 \cdot 10^{5}$ à $y / R=1$ (axe de la conduite), $y / R=0,105$ et $y / R=0,027$ (paroi). A titre de comparaison, la distribution normale de Gauss $(s=0 ; \gamma=3)$ est indiquée. 


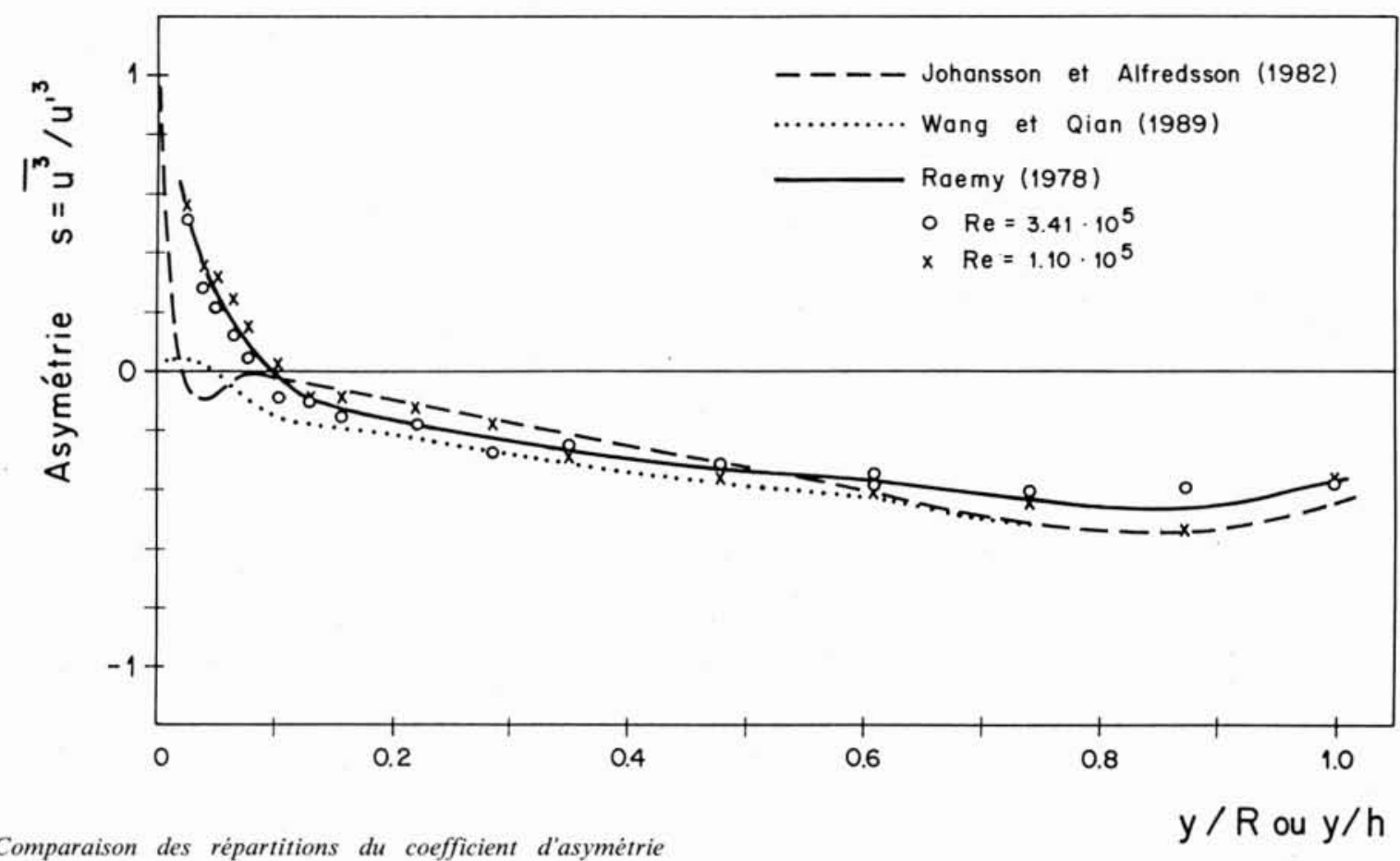

6. Comparaison des répartitions du coefficient d'asymétrie $s$ obtenues dans divers écoulements d'eau.

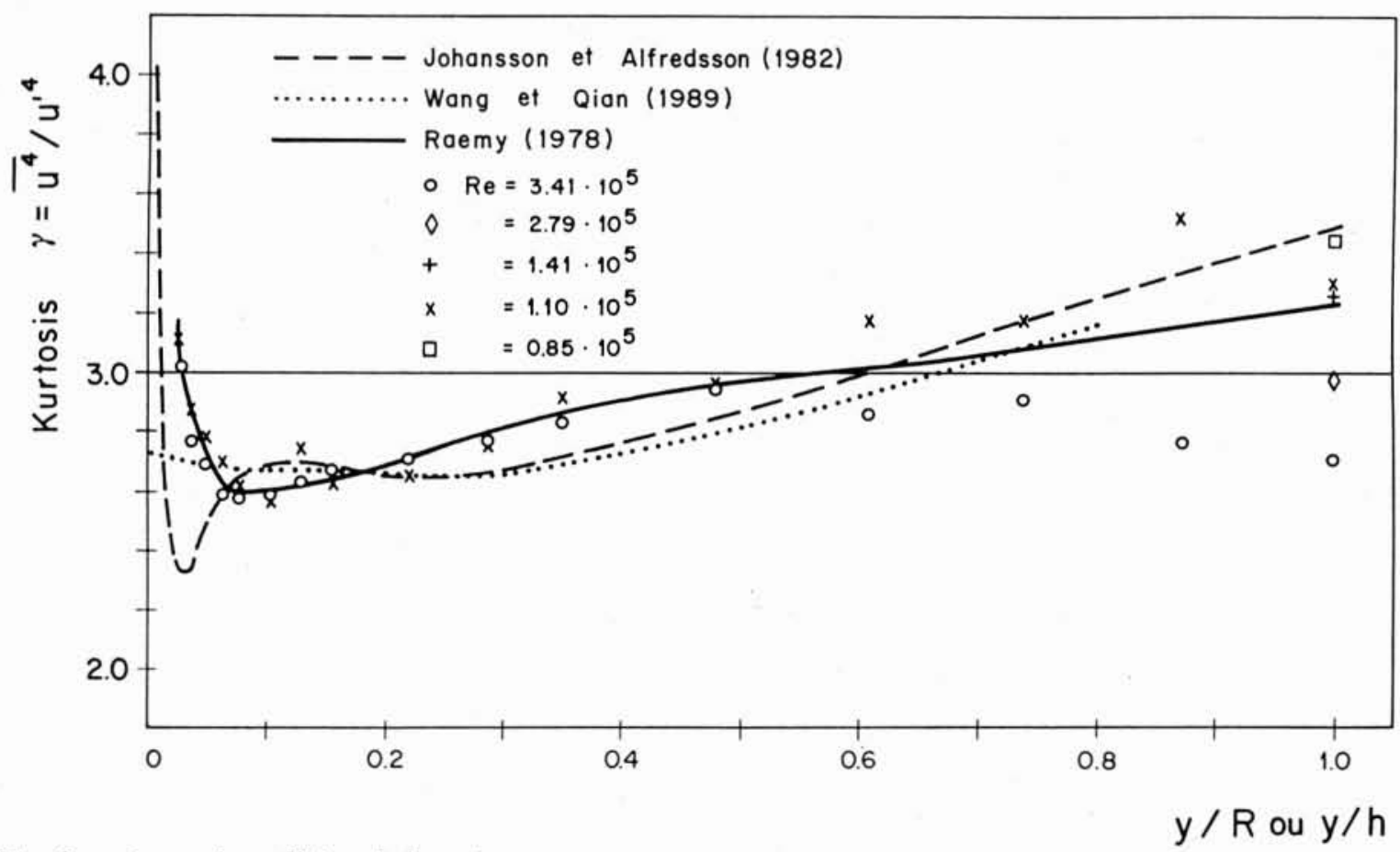

7. Dito figure 6, pour le coefficient de kurtosis $\gamma$.

Comme pour les essais effectués aux autres Re, il est visible que l'asymétrie des distributions est positive au voisinage de la paroi et négative au centre de la conduite. Une telle constatation avait aussi été faite par GRASs [7] en canal lisse et rugueux, de même que par Durst [6] dans un écoulement d'air en conduite lisse. Sur les figures 6 et 7 , les répartitions des coefficients $s$ et $\gamma$ dans la conduite rugueuse sont comparés aux résultats obtenus ultérieurement dans des écoulements d'eau sur fond lisse (JohansSON et AlfredsSON [8]; WANG et QIAN [22]). 


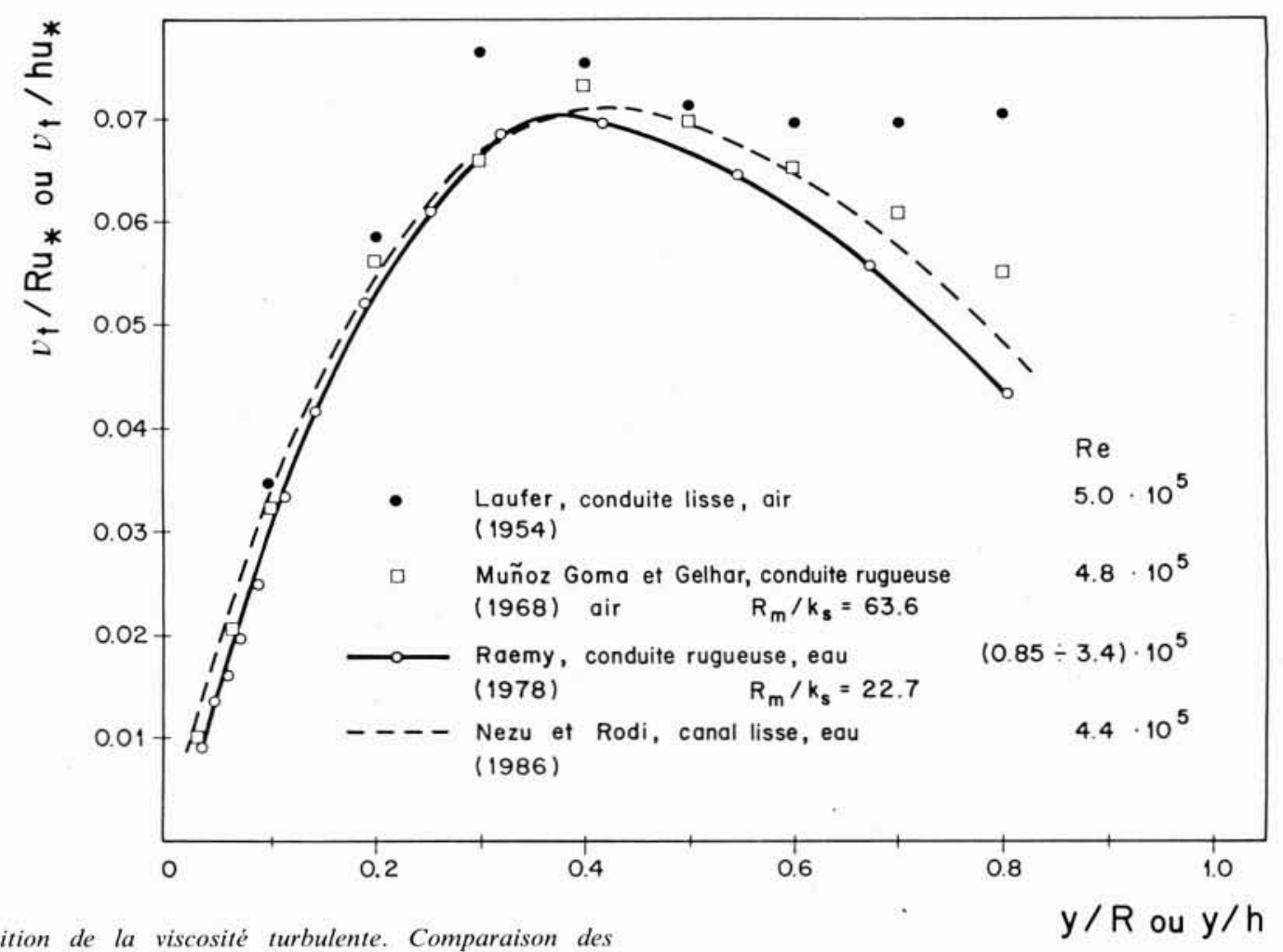

8. Répartition de la viscosité turbulente. Comparaison des résultats obtenus en conduite lisse et rugueuse, respectivement en canal lisse.



. Répartitions de la microéchelle de Taylor dans divers écoulements. 
Hormis dans la zone proche de la paroi $(y / R$ ou $y / h \leqslant 0,1$ ), les diverses répartitions de l'asymétrie coïncident entre elles et aucune dépendance marquée du nombre de Reynolds n'est décelable. Une constatation identique peut être faite au sujet de la kurtosis $\gamma$ malgré une certaine dispersion des valeurs au centre de la conduite rugueuse. En aucun point de l'écoulement, la condition $s=0$ et $\gamma=3$ propre à une répartition gaussienne ne paraît être remplie.

Les récents résultats obtenus dans un écoulement d'eau sur fond lisse cités ici semblent ainsi confirmer ceux relevés antérieurement dans la conduite rugueuse.

\section{Viscosité turbulente}

La répartition de la viscosité turbulente $v_{t}$ a été calculée dans la conduite rugueuse à partir du gradient des profils de vitesse moyenne locale mesurés. Avec

$$
\frac{\tau}{\rho}=v_{t} \cdot \frac{\mathrm{d} \bar{U}}{\mathrm{~d} y}
$$

où

$\tau=$ contrainte tangentielle

$\rho=$ masse spécifique de l'eau

$\bar{U}=$ vitesse moyenne locale

et en admettant la distribution linéaire

$$
\frac{\tau}{\rho}=\frac{\tau_{0}}{\rho}\left(1-\frac{y}{R}\right)=u^{2} \cdot\left(1-\frac{y}{R}\right)
$$

on a pour $v_{t}$ la forme adimensionnelle

$$
\frac{v_{t}}{u \cdot \cdot R}=\frac{u \cdot}{\bar{U}_{\max }} \cdot \frac{1-\xi}{\frac{\mathrm{d}}{\mathrm{d} \xi}\left(\frac{\bar{U}}{\bar{U}_{\max }}\right)}
$$

où $\bar{U}_{\max }=$ vitesse moyenne sur l'axe de la conduite

$$
\xi=y / R .
$$

Dans le domaine du frottement rugueux étudié, toutes les répartitions $\bar{U} / \bar{U}_{\max }=f(y / R)$ et le rapport $u \cdot / \bar{U}_{\max }$ étant indépendants de $\mathrm{Re}$, les valeurs $v_{t} /(u \cdot R)$ données dans la figure 8 pour l'écoulement d'eau en conduite rugueuse (RAEMY [14]) sont valables pour tous les essais réalisés. Ces résultats sont comparés à ceux de LAUFER [9] en conduite lisse, à ceux de MuÑoz GoMA et GELHAR [10] en conduite rugueuse et à ceux de NEZU et RODI [12] en canal découvert à fond lisse.

On remarque qu'au voisinage de la paroi, les diverses répartitions coïncident, $v_{t}$ augmentant linéairement avec $y / R$ ou $y / h$. Après avoir atteint un maximum à $y / R \cong 0,4$, les valeurs $v_{t} /\left(u_{*} \cdot R\right)$ obtenues en conduite divergent. Sur paroi lisse, elles restent pratiquement constantes tandis que sur paroi rugueuse, elles diminuent progressivement en direction de l'axe. Cet effet augmente lorsque
$R_{m} / k_{s}$ décroît. En canal découvert, la courbe donnée par NEZU et RODI (fig. 8) tient compte du paramètre de sillage $\Pi$ introduit par COLES [5] et qui caractérise la répartition des vitesses dans la zone externe. De leurs mesures (voir [12]), ils proposent pour $\mathrm{Re} \geqslant 1.10^{5}$ la valeur constante $\Pi=0,2$ utilisée ici. Cette courbe est comparable à celles obtenues en conduite rugueuse pour $y / R \leqslant 0,8$. Elle s'en distingue toutefois à la surface libre de l'écoulement $(y / h=1)$ où $v_{t}$ devient nulle.

\section{Microéchelles de Taylor}

La microéchelle de Taylor, ou longueur de dissipation, peut être calculée à partir de la densité spectrale de puissance ou par dérivation directe du signal turbulent. Désignée par $\lambda_{x}$ en direction longitudinale, elle a été déterminée dans la conduite rugueuse (RAEMY [14]) selon cette dernière méthode avec

$$
\lambda_{x}=\bar{U} \cdot u^{\prime} \cdot \frac{1}{\sqrt{\overline{\left(\frac{\partial u}{\partial t}\right)^{2}}}}
$$

où $\partial u / \partial t$ est la dérivée du signal

Les répartitions de $\lambda_{x}$ obtenues dans la section de la conduite sont montrées pour trois $\mathrm{Re}$ à la figure 9. A rugosité relative donnée, on voit que $\lambda_{x}$ diminue lorsque $\mathrm{Re}$ augmente. Faibles à la paroi, les valeurs de la microéchelle augmentent jusqu'à un maximum situé à $y / R \cong 0,5 \div 0,6$. Les résultats de Powe et Townes [13], acquis dans l'air en conduite lisse et rugueuse, montrent qu'à $\operatorname{Re}$ fixé, $\lambda_{x}$ décroît avec l'augmentation de la rugosité.

L'allure générale des répartitions reste identique. Une constatation semblable peut être faite en canal à fond lisse (RAICHLEN [15], WANG et QIAN [22]) où $\lambda_{x}$ est maximum à $y / h \cong 0,4$. Comparant leurs résultats à ceux de différents travaux, ROBERTSON et autres [18] [19] avaient conclu que $\lambda_{x}$ est une valeur pratiquement constante égale à env. $0,3 \mathrm{~cm}$ dans l'air et à env. le double, soit $0,6 \mathrm{~cm}$, dans l'eau. Les informations contenues à la figure 9 ne permettent pas de confirmer cette observation. En effet, $\lambda_{x}$ varie entre $0,13 \mathrm{~cm}$ à la paroi et $0,32 \mathrm{~cm}$, resp. $0,42 \mathrm{~cm}$ à $y / R=0,6$ (RAEMY), entre $0,69 \mathrm{~cm}$ et $1,38 \mathrm{~cm}$ à $y / h=0,4$ (RAICHLEN), et pour les essais de POwE et Townes, de $0,6 \mathrm{~cm}$ à $1,44 \mathrm{~cm}$ en conduite lisse, resp. de $0,6 \mathrm{~cm}$ à $1,2 \mathrm{~cm}$ en conduite rugueuse. Les valeurs absolues de $\lambda_{x}$ mesurées par WANG et QIAN n'ont pu être retrouvées, les paramètres hydrauliques de l'essai présenté n'étant pas indiqués dans leur publication. Il convient de rappeler ici que la précision de la détermination numérique des microéchelles est fortement influencée par le choix de la fréquence d'échantillonnage $f_{s}$ et de la fréquence de coupure $f_{\max }$ du signal turbulent. Si ces fréquences sont choisies trop basses, une perte importante d'information par lissage du signal se produit et $\lambda_{s}$ subit une erreur d'estimation par excès (RAEMY [14]). 


\section{Conclusions}

Les résultats de récentes mesures de turbulence effectuées dans l'eau par différents auteurs sont comparés à ceux obtenus antérieurement dans une conduite circulaire à paroi rugueuse [14]. Les conclusions suivantes peuvent être tirées :

- Les diverses méthodes utilisées dans l'eau pour la mesure des vitesses instantanées locales (anémomètres à film chaud et à laser, vélocimètres) conduisent à des résultats présentant une bonne concordance. En particulier, les observations faites lors de l'étude en conduite rugueuse [14] sont, dans leur ensemble, confirmées.

- Les répartitions des intensités turbulentes normalisées par la vitesse de frottement sont, sauf dans la zone adjacente à la paroi $(y / R$ ou $y / h \leqslant 0,2)$, indépendantes du nombre de Reynolds et de la rugosité relative. Pour les valeurs $D=2,37$ et $\lambda=1,03$, la répartition obtenue dans la conduite rugueuse [14] coïncide de manière satisfaisante avec la répartition " universelle » proposée par NeZU [11].
- Les coefficients d'asymétrie $s$ et de la kurtosis $\gamma$ déterminés en canal découvert lisse, respectivement en conduite à section rectangulaire lisse, présentent une même distribution que dans la conduite rugueuse. La répartition de la densité de probabilité de la vitesse longitudinale n'est nulle part gaussienne dans le profil de mesure.

- Dans la conduite rugueuse [14], la répartition de la viscosité turbulente $v_{t} /\left(u_{*} \cdot R\right)$ est indépendante de Re et suit pratiquement celle trouvée par NEZU et RODI [12] en canal découvert lisse avec le paramètre de sillage $\Pi=0,2$ valable pour $\mathrm{Re} \geqslant 1 \cdot 10^{5}$.

- Les répartitions des microéchelles de Taylor $\lambda_{x}$ montrent un maximum situé approximativement à $y / R$ ou $y / h$ variant entre 0,4 et 0,6 . Les valeurs de $\lambda_{x}$ diminuent lorsque $\mathrm{Re}$ et la rugosité de paroi augmentent.

- L'ensemble des résultats présentés confirment qu'actuellement la turbulence peut être mesurée dans l'eau avec une qualité comparable à celle obtenue dans l'air.

Symboles

D constante empirique

$D_{m} \quad$ diamètre moyen de la conduite rugueuse

$d_{m}^{m} \quad$ diamètre moyen des grains avant collage

$f_{\max }$ fréquence de Nyquist

$f_{s} \quad$ fréquence d'échantillonnage

$h$ profondeur d'écoulement

$k_{s} \quad$ hauteur d'aspérité équivalente de la rugosité

$p(U)$ densité de probabilité de la vitesse

$\hat{p}(U)$ estimateur de $p(U)$

$R \quad$ rayon de la conduite rugueuse rapporté à l'origine de $y$; rayon en conduite lisse

$\operatorname{Re}$ nombre de Reynolds $\operatorname{Re}=\bar{U}_{p} \cdot D_{m} / \nu$ ou $\operatorname{Re}=\bar{U}_{p} \cdot 4 R_{h} / \nu$

$R_{h} \quad$ rayon hydraulique

$s \quad$ coefficient d'asymétrie

$t$ temps

$U$ vitesse instantanée locale $(U=\bar{U}+u)$

$\bar{U} \quad$ vitesse moyenne locale

$\bar{U}_{p} \quad$ vitesse de débit $u \quad$ composante axiale (longitudinale) de la fluctuation de vitesse

$u^{\prime} \quad$ écart-type de la fluctuation $u$ de la vitesse (intensité turbulente)

u. vitesse de frottement $\left(u \cdot=\sqrt{\tau_{0} / \rho}\right)$

$y$ distance à la paroi (conduite) ou sur le fond du canal

$z \quad$ abscisse en représentation adimensionnelle de la densité de probabilité $\left(z=u / u^{\prime}\right)$

coefficient de kurtosis

constante empirique

microéchelle de Taylor

viscosité cinématique

viscosité turbulente

distance réduite à la paroi $(\xi=y / R)$

paramètre de sillage de Coles

masse spécifique du fluide

contrainte tangentielle

contrainte tangentielle à la paroi 


\section{Références bibliographiques}

[1] Blinco, P. H. and Partheniades, E. : "Turbulence Characteristics in Free Surface Flows over Smooth and Rough Boundaries ". Journal of Hydraulic Research, Vol. 9, No. 1, 1971, pp. 43-69.

[2] BLinco, P. H. and Simons, D. B. : "Characteristics of Turbulent Boundary Shear Stress ", Journal of the Engineering Mechanics Division, ASCE, Vol. 100, No. EM2, Proc. Paper 10477, 1974, pp. 203-220.

[3] Cardoso, A. H., Gust, G., Graf, W. H. : " Uniform Flow in a Smooth Open Channel ", Journal of Hydraulic Research, Vol. 27, No. 5, 1989, pp. 603-616.

[4] Clark, J. A. : "A Study of Incompressible Turbulent Boundary Layers in Channel Flow ", Transactions of the ASME, Journal of Basic Engineering, 1968, pp. 455-467.

[5] Coles, D. : "The Young Person's Guide Data", Proc., AFOSR-IFP Standford Conference on Computation of Turbulent Boundary Layers, edited by Coles \& Hirst, Vol. 2, 1968.

[6] Durst, F.: "10 LDA Application Notes", edited by Durst F., Karlsruhe, 1975 and published by DISA Information Dept., Publ. No. 6202 E, 1976, pp. 27-28.

[7] Grass, A. J. : «Structural Features of Turbulent Flow over Smooth and Rough Boundaries ", Journal of Fluid Mechanics, Vol. 50, part 2, pp. 233-255, 1971.

[8] Johansson, A. V. and Alfredsson, P. H. : "On the Structure of Turbulent Channel Flow ", Journal of Fluid Mechanics, Vol. 122, pp. 295-314, 1982.

[9] LAUfer, J. : "The Structure of Turbulence in Fully Developed Pipe Flow n, NACA, report 1174, 1954.

[10] Munoz Goma, R. J. and Gelhar, L. W., "Turbulent Pipe Flow with Rough and Porous Walls ", MIT, Hydrodynamics Laboratory, Report No. 109, 1968.

[11] Nezu, 1. : "Turbulent Structure in Open-Channel Flows", $\mathrm{Ph}$. D. Thesis, Kyoto University, Japan, 1977.

[12] Nezu, I. and Rodi, W. : "Open-Channel Flow Measurements with a Laser Doppler Anemometer ", Journal of Hydraulic Engineering, ASCE, Vol. 112, No. 5, 1986, pp. 335-355.
[13] Powe, R. E. and Townes, H. W. : "Turbulence Structure for Fully Developed Flow in Rough Pipes ", Transactions of the ASME, Journal of Fluid Engineering, No. 6, 1973, pp. 255-262.

[14] RAEMY, F. : "Mesure de la turbulence dans un écoulement d'eau en conduite circulaire à paroi rugueuse". Thèse, Institut d'Hydromécanique et d'Aménagement des Eaux, EPF, Zurich, R 14-78, 1978.

[15] Raichlen, F. : "Some Turbulence Measurements in Water ", Journal of the Engineering Merhanics Division, ASCE, Vol. 93, No. EM2, Proc. Paper 5195, 1967, pp. 7397.

[16] ReSCH, F.: "Etudes sur le fil chaud et le film chaud dans l'eau ", Thèse Doct. Ing., Marseille, 1968.

[17] Richardson, E. V. and McQuivey, R. S. : "Measurement of Turbulence in Water ", Journal of the Hydraulics Division, ASCE, Vol. 94, No. HY2, Proc. Paper 5855, 1968, pp. 411-429.

[18] Robertson, J. M., Burkhart, T. H., Martin, J. D. : " A Study of Turbulent Flow in Rough Pipes", University of Illinois, T \& AM Report, No. 279, 1965.

[19] Robertson, J. M. : " Some Turbulence Measurements in Water ", Discussion du travail de Raichlen, F. (voir [15]), Journal of the Eng. Mech. Division, ASCE. No. EM6, 1967.

[20] Steffler, P. M., Rajaratnam, N., Peterson, A. W. «LDA Measurements of Mean Velocity and Turbulence Distribution in a Smooth Rectangular Open Channel", Water Ressources Engineering, Report 83-4, Dept. of Civil Eng., University of Alberta, Canada, 1983.

[21] Townes, H. W., Gow, J. L., Powe, R. E., Weber, N. : "Turbulent Flow in Smooth and Rough Pipes ", Trans. of the ASME, Vol. 94, Series D, No. 2, 1972, pp. 353-362.

[22] WANG, X. and QIAN, N. : "Turbulent Characteristics of Sediment-Laden Flow ", Journal of Hydraulic Engineering. ASCE, Vol. 115, No. 6, 1989, pp. 781-800. 